\title{
Interlayer Coherent Composite Fermi Liquid Phase in Quantum Hall Bilayers
}

\author{
Jason Alicea, ${ }^{1}$ Olexei I. Motrunich, ${ }^{1}$ G. Refael, ${ }^{1}$ and Matthew P. A. Fisher ${ }^{2}$ \\ ${ }^{1}$ Department of Physics, California Institute of Technology, Pasadena, California 91125, USA \\ ${ }^{2}$ Microsoft Research, Station Q, University of California, Santa Barbara, California 93106, USA
}

(Received 26 August 2009; published 17 December 2009)

\begin{abstract}
We introduce an interlayer coherent composite Fermi liquid for $\nu=1 / 2+1 / 2$ bilayers, in which interlayer Coulomb repulsion drives exciton condensation of composite fermions. As a result, composite fermions propagate coherently between layers — even though electrons do not-and form bonding and antibonding Fermi seas. This phase is compressible with respect to symmetric currents but quantum Halllike in the counterflow channel. Quantum oscillations of the composite Fermi seas generate a new series of incompressible states at $\nu=p /[2(p \pm 1)]$ per layer ( $p$ an integer), which is a bilayer analogue of Jain's sequence.
\end{abstract}

DOI: 10.1103/PhysRevLett.103.256403

Composite fermions (CF's) have played a central role in the field of quantum Hall physics [1]. Perhaps the most striking manifestation of CF's is their formation of a Fermi sea at certain even-denominator fillings, notably $\nu=1 / 2$. Pioneering work by Halperin, Lee, and Read (HLR) developed the theory of such composite Fermi liquids (CFL's) [2], and the anticipated Fermi surface has been experimentally measured $[1,3]$. As a corollary of this interactiondriven "metallicity," CFL's provide a unified picture for Jain's sequence [1] - these quantum Hall states emerge as quantum oscillations of a composite Fermi sea [2].

Given the success of HLR theory, it is natural to inquire whether CFL's can emerge in strongly coupled multilayer systems. More precisely, can interlayer Coulomb repulsion generate coherent propagation of CF's between layers, resulting in an interlayer coherent CFL with a higherdimensional composite Fermi surface? Such a phase would constitute a fundamentally new kind of CFL and, if found, would broaden the utility of CF's into a new dimension. Experimentally, this question is motivated by quantum Hall bilayers, for which compressible states appear even when interlayer repulsion is "strong" (e.g., [4]). Additionally, experiments on bismuth [5] highlight our lack of understanding of strongly interacting threedimensional systems in the lowest Landau level (LLL), further stimulating the quest for exotic multilayer phases.

Here we argue that spin-polarized bilayers at $\nu=1 / 2$ per layer may indeed harbor an interlayer coherent CFL when the layer spacing $d$ and magnetic length $\ell_{B}$ are comparable (see Fig. 1). To motivate this phase, let us recall the well-understood physics at extreme $d / \ell_{B}$. For $d / \ell_{B} \lesssim 1$, interlayer Coulomb repulsion drives exciton condensation of the electrons [6], $\left\langle c_{\uparrow}^{\dagger} c_{\downarrow}\right\rangle \neq 0$, with $c_{\alpha}$ the electron operator in layer $\alpha=\uparrow, \downarrow$. When $d / \ell_{B} \rightarrow \infty$, the layers decouple and form $\nu=1 / 2$ CFL's with independent Fermi surfaces in each layer as in Fig. 2(a). Here the wave function is $\psi_{\infty}=P_{\mathrm{LLL}} \prod_{i<j}\left(z_{i}-z_{j}\right)^{2}\left(w_{i}-w_{j}\right)^{2} \Psi_{\mathrm{CF}}^{\uparrow} \Psi_{\mathrm{CF}}^{\downarrow}$, where $P_{\text {LLL }}$ denotes LLL projection, $z, w$ are coordinates in layer $\uparrow, \downarrow$, and $\Psi_{\mathrm{CF}}^{\alpha}$ is the composite Fermi sea wave
PACS numbers: 71.10.Pm, 73.21.Ac, 73.43.Cd

function in layer $\alpha$. The behavior at intermediate $d / \ell_{B}$ is far subtler and has been actively studied for more than a decade (for a recent discussion, see [7]).

We propose that for intermediate $d / \ell_{B}$, the short-range part of the interlayer Coulomb repulsion naturally favors exciton condensation of CF's, which generates an interlayer coherent CFL. Denoting the CF operator by $f_{\alpha}$, this phase is characterized by $\left\langle f_{\uparrow}^{\dagger} f_{\downarrow}\right\rangle \neq 0$ even though $\left\langle c_{\uparrow}^{\dagger} c_{\downarrow}\right\rangle=$ 0 . It follows that CF's (but not electrons) spontaneously tunnel between layers and form bonding and antibonding composite Fermi surfaces as in Fig. 2(b). A simple trial wave function for this new phase is

$$
\psi=P_{\mathrm{LLL}} \prod_{i<j}\left(z_{i}-z_{j}\right)^{2}\left(w_{i}-w_{j}\right)^{2} \Psi_{\left(k_{F, B}, k_{F, A}\right)},
$$

where $k_{F, B(A)}$ is the bonding (antibonding) Fermi momentum and the determinant $\Psi_{\left(k_{F, B}, k_{F, A}\right)}$ fills the Fermi seas. While the interlayer coherent CFL behaves similarly to decoupled CFL's in response to symmetric currents, in the counterflow channel it behaves like a $\nu=1 / 2$ quantum Hall state. This follows from CF exciton condensation, just as electron exciton condensation leads to counterflow superfluidity at small $d / \ell_{B}$ [6]. Interestingly, quantum oscillations of the Fermi surfaces generate a bilayer analogue of Jain's sequence [see Eqs. (8) and (9)], which includes the 331 state [8] and other observed fractions.

Although universal features will be our main focus, we will argue that the interlayer coherent CFL will (at least) be energetically competitive in a model with spin-polarized

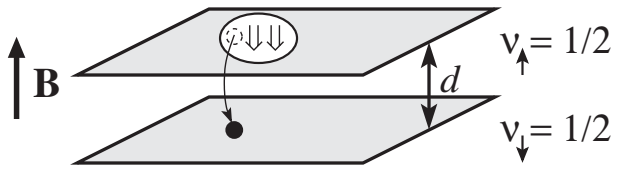

FIG. 1. Schematic of bilayer setup. The electron is a bound state of two flux quanta and a composite fermion (shown by the small circles). In the interlayer coherent CFL, composite fermions tunnel between layers, while electrons do not. 

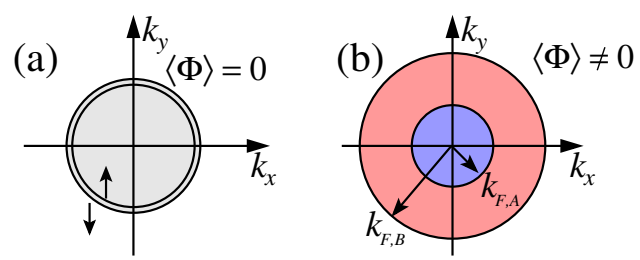

FIG. 2 (color online). (a) At $d / \ell_{B}=\infty$ and $\nu=1 / 2$ per layer, composite fermions form decoupled CFL's with identical Fermi surfaces in the $\uparrow$ and $\downarrow$ layers. (b) At intermediate $d / \ell_{B}$, we propose an interlayer coherent CFL where composite fermions coherently tunnel between layers and form bonding and antibonding Fermi surfaces with radii $k_{F, B}$ and $k_{F, A}$.

electrons and zero interlayer tunneling, and thus constitutes a serious candidate phase. Consider then the Euclidean action

$$
S=\int_{x} \sum_{\alpha=\uparrow, \downarrow} c_{x \alpha}^{\dagger}\left[\partial_{\tau}-\frac{(\nabla+i e \mathbf{A})^{2}}{2 m}\right] c_{x \alpha}+S_{\text {Coul }},
$$

where $x=(\mathbf{r}, \tau), \mathbf{B}=\nabla \times \mathbf{A}$ is the external field, and $S_{\text {Coul }}=S_{\text {Coul }}^{\Uparrow}+S_{\text {Coul }}^{\Downarrow}+S_{\text {Coul }}^{\uparrow \downarrow}$ encodes the Coulomb repulsion. (We set $\hbar=c=1$.) We focus primarily on fillings $\nu_{\uparrow}=\nu_{\downarrow}=1 / 2$ at intermediate $d / \ell_{B}$, such that the exciton condensate is destroyed but interlayer Coulomb is not weak. We postulate that CF's remain the "correct" degrees of freedom here and write the action as

$$
\begin{aligned}
S_{\mathrm{CF}}= & \int_{x} \sum_{\alpha=\uparrow, \downarrow}\left\{f_{x \alpha}^{\dagger}\left[\left(\partial_{\tau}-i a_{\alpha}^{0}\right)-\frac{\left(\nabla-i \mathbf{a}_{\alpha}\right)^{2}}{2 m^{*}}\right] f_{x \alpha}\right. \\
& \left.+\frac{i}{8 \pi}\left(a_{\alpha}^{\mu}+e \mathcal{A}_{\alpha}^{\mu}\right) \epsilon_{\mu \nu \lambda} \partial_{\nu}\left(a_{\alpha}^{\lambda}+e \mathcal{A}_{\alpha}^{\lambda}\right)\right\}+S_{\mathrm{Coul}} .
\end{aligned}
$$

Here $m^{*}$ is the CF mass [2], $a_{\alpha}^{\mu}$ denotes the Chern-Simons fields, and $\mathcal{A}_{\alpha}^{\mu}=A^{\mu}+\delta A_{\alpha}^{\mu}$, with $\delta A_{\alpha}^{\mu}$ a probe field added for computing response properties. The ChernSimons term above attaches two flux quanta to each CF, recovering the physical electron as in Fig. 1.

Equation (3) was previously studied in important work by Bonesteel et al. [9] By examining the effect of longwavelength gauge fluctuations at $d / \ell_{B} \gg 1$, these authors argued that such coupled CFL's should generically undergo an interlayer BCS pairing instability. This is rather natural at large $d / \ell_{B}$ within the dipole picture of decoupled CFL's [10]. However, when $d / \ell_{B}$ is of order unity the layers are strongly coupled, so here one should first attack the problem by satisfying the short-distance, high-energy physics.

To this end, we focus on the interlayer Coulomb $S_{\text {Coul }}^{\uparrow \downarrow}$, decomposed into short-range and long-range pieces via $S_{\mathrm{Coul}}^{\uparrow \downarrow}=S_{\mathrm{sr}}^{\dagger \downarrow}+S_{\mathrm{lr}}^{\dagger \downarrow}$. The short-range part can be written

$$
S_{\mathrm{sr}}^{\uparrow \downarrow}=u \int_{x} f_{x \uparrow}^{\dagger} f_{x \downarrow}^{\dagger} f_{x \downarrow} f_{x \uparrow}=-u \int_{x} f_{x \uparrow}^{\dagger} f_{x \downarrow} f_{x \downarrow}^{\dagger} f_{x \uparrow} ;
$$

including interactions out to a range $\ell_{B}$, we crudely estimate $u \approx\left(e^{2} / d\right)\left(\pi \ell_{B}^{2}\right)$. Short-range interlayer Coulomb is thus attractive in the particle-hole rather than the Cooper channel, and favors $\mathrm{CF}$ exciton condensation. To expose this competing instability, we decouple Eq. (4) with a Hubbard-Stratonovich field $\Phi$, which can be regarded as a $\mathrm{CF}$ exciton condensate order parameter:

$$
\begin{aligned}
S_{\mathrm{sr}}^{\uparrow \downarrow} \rightarrow & \int_{x}\left[\frac{1}{u}|\Phi(x)|^{2}-\left(f_{x \uparrow}^{\dagger} f_{x \downarrow} \Phi(x)+\text { H.c. }\right)\right. \\
& \left.+\kappa\left|\left[\partial_{\mu}-i\left(a_{\uparrow}^{\mu}-a_{\downarrow}^{\mu}\right)\right] \Phi(x)\right|^{2}\right] .
\end{aligned}
$$

We include a kinetic term for $\Phi$ here, which minimally couples to $a_{\uparrow}^{\mu}-a_{\downarrow}^{\mu}$ to maintain gauge invariance.

When $u$ exceeds a critical value, $\Phi$ condenses and the system enters an interlayer coherent CFL phase (Ref. [11] predicts a similar instability for electrons at $B=0$ ). To estimate when this transpires, we integrate out the CF's to derive an effective theory for $\Phi$ coupled to $a_{\uparrow}^{\mu}-a_{\downarrow}^{\mu}$. To leading order, the coefficient of $|\Phi(x)|^{2}$ shifts to $\frac{1}{u}-\frac{m^{*}}{2 \pi}$. Using our earlier estimate for $u$, this vanishes when $\left(d / \ell_{B}\right)_{c} \approx e^{2} \ell_{B} m^{*} / 2$, which is just the Stoner criterion for "pseudospin ferromagnetism" of CF's. (CF ferromagnetism has previously been discussed for single-layers with spin in the context of incompressible states [1].) Inserting $1 / m^{*} \approx e^{2} \ell_{B} / 6$ from Ref. [12] yields $\left(d / \ell_{B}\right)_{c} \approx 3$. Ultimately, however, $\left(d / \ell_{B}\right)_{c}$ should be determined numerically as we discuss below.

As an aside, we comment on the case with $\nu_{\uparrow, \downarrow}=1 / 4$, where CF's have an effective filling $\nu_{\uparrow, \downarrow}^{\mathrm{CF}}=1 / 2$. From Eqs. (4) and (5) one similarly expects interlayer Coulomb to drive exciton condensation of CF's below a critical layer separation. CF's then form the 111 state, so the electron wave function is $\psi=\prod_{i<j}\left(z_{i}-z_{j}\right)^{2} \times$ $\left(w_{i}-w_{j}\right)^{2} \Psi_{111}$, i.e., the 331 state. There is strong experimental $[13,14]$ and theoretical [15-17] evidence that this phase indeed emerges at intermediate $d / \ell_{B}$, which substantiates the above logic.

We now return to $\nu_{\uparrow, \downarrow}=1 / 2$ and characterize the interlayer coherent CFL with $\langle\Phi\rangle \neq 0$. The first remarkable property of this phase, which follows from Eq. (5), is that CF's are liberated from their layers and coherently interlayer tunnel. Consequently, bonding and antibonding composite Fermi seas form as in Fig. 2(b). The respective Fermi momenta $k_{F, B}$ and $k_{F, A}$ are determined by $d / \ell_{B}$ but satisfy $k_{F, B}^{2}+k_{F, A}^{2}=2 / \ell_{B}^{2}$ to yield the correct filling. In contrast, at $d / \ell_{B}=\infty \mathrm{CF}$ 's are confined to their layers and form equal-size Fermi surfaces as in Fig. 2(a).

Crucially, although CF's tunnel, electrons do not. This can be seen by reformulating the problem using Wen's parton construction [18], expressing the electron as $c_{\mathbf{r} \alpha}=$ $b_{\mathbf{r} \alpha} f_{\mathbf{r} \alpha}$. Here $b_{\mathbf{r} \alpha}$ is a boson that mimics the flux attachment and $f_{\mathbf{r} \alpha}$ is the CF; to remain in the physical Hilbert space one imposes the local constraint $c_{\mathbf{r} \alpha}^{\dagger} c_{\mathbf{r} \alpha}=b_{\mathbf{r} \alpha}^{\dagger} b_{\mathbf{r} \alpha}=$ $f_{\mathbf{r} \alpha}^{\dagger} f_{\mathbf{r} \alpha}$. In the interlayer coherent CFL, CF's tunnel coherently but the bosons form decoupled $\nu=1 / 2$ quantum Hall states in each layer. Because of the constraint, electron tunneling requires both $b_{\mathbf{r} \alpha}$ and $f_{\mathbf{r} \alpha}$ to tunnel, but only the latter can do so as Fig. 1 illustrates. 
Partons also allow correlations to be estimated in a mean-field approximation that neglects the Hilbert-space constraint. Consider the interlayer density-density correlation function $g_{\uparrow \downarrow}\left(\mathbf{r}-\mathbf{r}^{\prime}\right)=\rho^{-2}\left\langle c_{\mathbf{r} \uparrow}^{\dagger} c_{\mathbf{r}^{\prime} \downarrow}^{\dagger} c_{\mathbf{r}^{\prime} \downarrow} c_{\mathbf{r} \uparrow}\right\rangle$, where $\rho=$ $1 /\left(4 \pi \ell_{B}^{2}\right)$. Upon introducing partons and ignoring the constraint, $g_{\uparrow \downarrow}$ factorizes: $g_{\uparrow \downarrow}\left(\mathbf{r}-\mathbf{r}^{\prime}\right)=\rho^{-2}\left\langle b_{\mathbf{r} \uparrow}^{\dagger} b_{\mathbf{r}^{\prime} \downarrow}^{\dagger} b_{\mathbf{r}^{\prime} \downarrow} b_{\mathbf{r} \uparrow}\right\rangle \times$ $\left\langle f_{\mathbf{r}^{\dagger} \uparrow}^{\dagger} f_{\left.\mathbf{r}^{\prime}\right\rfloor}^{\dagger} f_{\left.\mathbf{r}^{\prime}\right\rfloor} f_{\mathbf{r}^{\dagger} \uparrow}\right\rangle$. The boson part yields a constant since the exchange term vanishes. Setting this contribution to unity (i.e., using "renormalized mean-field theory") yields $g_{\uparrow \downarrow}\left(\mathbf{r}-\mathbf{r}^{\prime}\right) \rightarrow \rho^{-2}\left\langle f_{\mathbf{r} \uparrow}^{\dagger} f_{\mathbf{r}^{\prime} \downarrow}^{\dagger} f_{\mathbf{r}^{\prime} \downarrow} f_{\mathbf{r}^{\prime} \uparrow}\right\rangle$, which evaluates to

$$
g_{\uparrow \downarrow}(\mathbf{r})=1-\frac{\ell_{B}^{4}}{r^{2}}\left[k_{F, B} J_{1}\left(k_{F, B} r\right)-k_{F, A} J_{1}\left(k_{F, A} r\right)\right]^{2} .
$$

Figure 3 displays $g_{\uparrow \downarrow}$ for several values of $k_{F, A} / k_{F, B}$, and demonstrates that reducing $k_{F, A} / k_{F, B}$ lowers the interlayer Coulomb energy by smoothly binding an interlayer correlation hole. Interestingly, this crude treatment already captures the oscillations in $g_{\uparrow \downarrow}$ found numerically in exact diagonalization ([19]; Fig. 10(b) in [7]) and DMRG [20].

In contrast to the electron exciton condensate at small $d / \ell_{B}$ which spontaneously breaks a physical $U(1)$ symmetry, the $\mathrm{CF}$ exciton condensate only breaks gauge symmetry. This follows from Eq. (5), where condensing $\Phi$ yields a mass term for $a_{\uparrow}^{\mu}-a_{\downarrow}^{\mu}$, thereby pinning the Chern-Simons fields for the two layers together. Interesting physical consequences follow, which we now discuss.

Electromagnetic response properties are most clearly organized in a basis of symmetric/antisymmetric currents $j_{s / a}^{\mu}=\frac{1}{\sqrt{2}}\left(j_{\uparrow}^{\mu} \pm j_{\downarrow}^{\mu}\right)$. The response of $j_{s / a}^{0}$ yields the compressibility with respect to symmetric density changes $\left(\nu_{\uparrow, \downarrow}=1 / 2+\delta \nu\right)$, and layer imbalance $\left(\nu_{\uparrow}=1 / 2+\delta \nu\right.$, $\nu_{\downarrow}=1 / 2-\delta \nu$ ). Spatial components $\vec{j}_{s / a}$, corresponding to symmetric and counterflow currents, respond to electric fields through $\vec{E}_{s / a}=\overleftrightarrow{\rho}_{s / a} \vec{j}_{s / a}$, with $\vec{E}_{s / a}=\frac{1}{\sqrt{2}}\left(\vec{E}_{\uparrow} \pm \vec{E}_{\downarrow}\right)$. To evaluate the $q \rightarrow 0$, static compressibilities and resistivities, it suffices to simply set $a_{\uparrow}=a_{\downarrow} \equiv a$ since $a_{\uparrow}-a_{\downarrow}$ is massive. The $\mathrm{CF}$ action can then be written

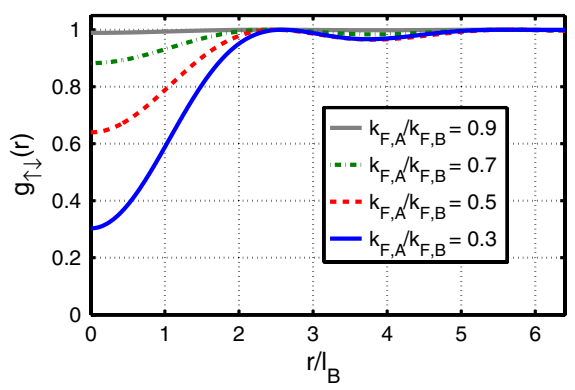

FIG. 3 (color online). Interlayer density-density correlation function in the interlayer coherent CFL computed in mean-field theory for various $k_{F, A} / k_{F, B}$. Reducing $k_{F, A} / k_{F, B}$ smoothly carves out an interlayer correlation hole as seen above.

$$
\begin{aligned}
S_{\mathrm{CF}} \rightarrow & \int_{x} \sum_{\alpha=\uparrow, \downarrow} f_{x \alpha}^{\dagger}\left[\left(\partial_{\tau}-i a^{0}\right)-\frac{(\nabla-i \mathbf{a})^{2}}{2 m^{*}}\right] f_{x \alpha} \\
& -t\left[f_{x \uparrow}^{\dagger} f_{x \downarrow}+\text { H.c. }\right]+S_{\text {Coul }}^{\uparrow}+S_{\text {Coul }}^{\Downarrow}+S_{\text {lr }}^{\uparrow \downarrow} \\
& +\int_{x}\left[\frac{i}{8 \pi}\left(\sqrt{2} a^{\mu}+e \mathcal{A}_{s}^{\mu}\right) \epsilon_{\mu \nu \lambda} \partial_{\nu}\left(\sqrt{2} a^{\lambda}+e \mathcal{A}_{s}^{\lambda}\right)\right. \\
& \left.+\frac{i e^{2}}{8 \pi} \mathcal{A}_{a}^{\mu} \epsilon_{\mu \nu \lambda} \partial_{\nu} \mathcal{A}_{a}^{\lambda}\right]
\end{aligned}
$$

with $t=\langle\Phi\rangle$ taken real and $\mathcal{A}_{s / a}^{\mu}=\frac{1}{\sqrt{2}}\left(\mathcal{A}_{\uparrow}^{\mu} \pm \mathcal{A}_{\downarrow}^{\mu}\right)$.

The Chern-Simons term for $\mathcal{A}_{a}$, which decouples from everything else, is the effective action for a $\nu=1 / 2$ fractional quantum Hall state. This has remarkable implications: despite having two Fermi surfaces, the interlayer coherent CFL behaves like an incompressible, gapped fractional quantum Hall state in the counterflow channel, with resistivity $\rho_{a}^{x x}=0$ and $\rho_{a}^{x y}=2 h / e^{2}$. As noted by Stern and Halperin [21], compressibility of a CFL is intimately tied to gauge invariance. Incompressibility thus formally stems from the partial breaking of gauge symmetry due to $\Phi$ condensing. More physically, transferring electrons from one layer to the other requires creating a net difference in flux between the layers; with $a_{\uparrow}-a_{\downarrow}$ massive doing so requires overcoming an energy gap.

To study such excitations, consider the more general CF action, Eqs. (3) and (5). Elementary excitations in this channel are created by inserting a vortex in $\Phi$, which by a singular gauge transformation is equivalent to adding $\pi$ flux in $a_{\uparrow}$ and $-\pi$ flux in $a_{\downarrow}$. An electron consists of a CF bound to $4 \pi$ flux, so the quasiparticles are formed by dipoles carrying charge $e / 4$ in one layer and $-e / 4$ in the other. The gap for creating these dipoles sets the temperature scale below which $\rho_{a}^{x x}$ will appear activated.

Since the symmetric channel preserves gauge symmetry, the interlayer coherent CFL and decoupled CFL's behave similarly here. Both are compressible, with resistivity $\rho_{s}^{x y}=2 h / e^{2}$ and $\rho_{s}^{x x}=\rho_{\mathrm{CF}}^{x x}$, where $\rho_{\mathrm{CF}}^{x x}$ is the CF resistivity with disorder. This can be obtained following [2], or with partons using the Ioffe-Larkin rule [22].

Given the compressibility in this channel, it is interesting to ask how the system evolves when the field shifts to $B=B_{1 / 2} \pm|\delta B|$ so that $\nu_{\uparrow, \downarrow}=1 / 2 \mp|\delta \nu|$. The attached flux now only partially cancels the field, so the bonding and antibonding Fermi surfaces develop into Landau levels such that the effective CF filling is $\nu_{\uparrow, \downarrow}^{\mathrm{CF}}=2 \pi \rho /(e|\delta B|)=$ $\nu_{\uparrow, \downarrow} /(2|\delta \nu|)$. Incompressible phases arise whenever an integer number $p_{B}$ of bonding and $p_{A}$ of antibonding Landau levels are filled-i.e., when $\nu_{\uparrow, \downarrow}^{\mathrm{CF}}=\left(p_{B}+p_{A}\right) / 2$. The corresponding electron filling factors and LLL wave functions are

$$
\begin{gathered}
\nu_{\uparrow, \downarrow}=\frac{p_{A}+p_{B}}{2\left(p_{A}+p_{B} \pm 1\right)}, \\
\psi_{\left(p_{B}, p_{A}\right)}=P_{\mathrm{LLL}} \prod_{i<j}\left(z_{i}-z_{j}\right)^{2}\left(w_{i}-w_{j}\right)^{2} \Psi_{\left(p_{B}, p_{A}\right)},
\end{gathered}
$$


where $\Psi_{\left(p_{B}, p_{A}\right)}$ is the wave function for the $p_{B / A}$ filled bonding and antibonding CF Landau levels. This series of interlayer-correlated quantum Hall states constitutes a bilayer analogue of the Jain sequence [1], and notably includes even-denominator fractions. Just as the Jain sequence can be viewed as quantum oscillations of a singlelayer CFL [2], this bilayer series emerges naturally as quantum oscillations of the interlayer coherent CFL; consequently, appearance of these fractions can serve as indirect evidence for its existence.

These properties of the interlayer coherent CFL distinguish it from other proposals for intermediate $d / \ell_{B}$ at $\nu_{\uparrow, \downarrow}=1 / 2$. For example, the interlayer BCS paired state is incompressible in the symmetric and antisymmetric channels [10]. Experimentally, double quantum wells studied to date seem unlikely to harbor an interlayer coherent CFL - a by-product of the counterflow incompressibility is a large longitudinal drag $\sim \rho_{\mathrm{CF}}^{x x}$, which is not observed [4]. This could be due to disorder-induced local filling factor variations, which this phase abhors, or perhaps partial spin polarization [23]. Wide quantum wells appear more promising; higher mobilities are achievable, and quantum Hall states have been observed at several of the fractions predicted by Eq. (8) [24-26]. Most intriguingly, the $70 \mathrm{~nm}$ wide quantum well in Ref. [25] exhibits a rich set of quantum oscillations around the compressible phase at $\nu=1 / 2+1 / 2$. These oscillations visibly die out in a wider $80 \mathrm{~nm}$ well [25], which may signify the transition from an interlayer coherent CFL to decoupled CFL's. Further studies of these quantum oscillations, such as the dependence on density imbalance, will provide important clues to their origin. If they represent the new bilayer quantum Hall series we identify, then these states should be killed by such imbalance, whereas competing "charge transfer states" [27] would be strengthened. Although interlayer tunneling makes contacting the layers separately difficult here, measuring directly the unequal Fermi surfaces at $\nu=1 / 2+1 / 2$ [3] provides another method of identifying this phase.

Our work opens up many other exciting future directions. One virtue of our proposal is the simplicity of the trial wave function in Eq. (1), which has one variational parameter. Thus, variational Monte Carlo calculations can be employed for large particle numbers to, e.g., compute $\left(d / \ell_{B}\right)_{c}$ below which CF's exciton condense and evaluate trial energies in model and realistic Hamiltonians. Comparison with exact diagonalization may also prove fruitful. One can further consider embellishments such as BCS pairing of one or both Fermi seas (yielding a nonAbelian state), finite-momentum pairing between the Fermi seas, and replacing CF's with composite bosons to smoothly connect with the 111 state at small $d / \ell_{B}$ [7]. Numerical studies of the new bilayer quantum Hall series emanating from this phase provide yet another important direction. These questions could be posed more generally at other fillings at which decoupled CFL's appear when $d / \ell_{B} \rightarrow \infty$ (e.g., $\left.\nu_{\uparrow, \downarrow}=1 / 4\right)$. Finally, a three-dimensional version of the bilayer coherent CFL—possibly relevant for layered semimetals like graphite-will be explored in future work.

It is a pleasure to acknowledge illuminating discussions with G. Chen, J. Eisenstein, Y.-B. Kim, D. Luhman, M. Milovanovic, G. Moller, and Y. Zou. We also acknowledge support from the Lee A. DuBridge Foundation (J. A), the A.P. Sloan Foundation (O. I. M), the Packard Foundation (G. R), and the NSF through Grants No. DMR- 0529399 (M.P.A.F), No. PHY-0456720, and No. PHY-0803371 (G. R).

[1] J. K. Jain, Phys. Rev. Lett. 63, 199 (1989); Composite Fermions (Cambridge University Press, London, 2007).

[2] B. I. Halperin P. A. Lee, and N. Read, Phys. Rev. B 47, 7312 (1993).

[3] Composite Fermions: A Unified View of the Quantum Hall Regime edited by O. Heinonen (World Scientific, Singapore, 1998).

[4] M. Kellogg et al., Phys. Rev. Lett. 90, 246801 (2003).

[5] K. Behnia, L. Balicas, and Y. Kopelevich, Science 317, 1729 (2007).

[6] Perspectives in Quantum Hall Effects edited by S. Das Sarma and A. Pinczuk (Wiley, New York, 1997).

[7] G. Möller, S. H. Simon, and E. H. Rezayi, Phys. Rev. B 79, 125106 (2009); Phys. Rev. Lett. 101, 176803 (2008).

[8] B. I. Halperin, Helv. Phys. Acta 56, 75 (1983).

[9] N.E. Bonesteel, I. A. McDonald, and C. Nayak, Phys. Rev. Lett. 77, 3009 (1996).

[10] Y. B. Kim et al., Phys. Rev. B 63, 205315 (2001).

[11] L. Zheng, M. W. Ortalano, and S. Das Sarma, Phys. Rev. B 55, 4506 (1997).

[12] R. Shankar and G. Murthy, Phys. Rev. Lett. 79, 4437 (1997).

[13] J. P. Eisenstein et al., Phys. Rev. Lett. 68, 1383 (1992).

[14] Y. W. Suen et al., Phys. Rev. Lett. 72, 3405 (1994).

[15] D. Yoshioka, A. H. MacDonald, and S. M. Girvin, Phys. Rev. B 39, 1932 (1989).

[16] S. He et al., Phys. Rev. B 43, 9339 (1991).

[17] K. Nomura and D. Yoshioka, J. Phys. Soc. Jpn. 73, 2612 (2004).

[18] X.-G. Wen, Phys. Rev. B 60, 8827 (1999).

[19] K. Nomura and D. Yoshioka, Phys. Rev. B 66, 153310 (2002).

[20] N. Shibata and D. Yoshioka, J. Phys. Soc. Jpn. 75, 043712 (2006).

[21] B. I. Halperin and A. Stern, Phys. Rev. Lett. 80, 5457 (1998).

[22] L. B. Ioffe and A. I. Larkin, Phys. Rev. B 39, 8988 (1989).

[23] I. B. Spielman et al., Phys. Rev. Lett. 94, 076803 (2005).

[24] M. Shayegan et al., Semicond. Sci. Technol. 11, 1539 (1996).

[25] D. Luhman et al., Physica (Amsterdam) 40E, 1059 (2008).

[26] D. R. Luhman et al., Phys. Rev. Lett. 101, 266804 (2008).

[27] H. C. Manoharan et al., Phys. Rev. Lett. 79, 2722 (1997). 